\title{
Anti-Biofilm Effect of Octenidine and Polyhexanide on Uropathogenic Biofilm-Producing Bacteria
}

\author{
Maria Loose ${ }^{\mathrm{a}} \quad$ Kurt G. Naber ${ }^{\mathrm{b}}$ Larry Purcell ${ }^{\mathrm{c}}$ Manfred P. Wirth ${ }^{\mathrm{d}}$ Florian \\ M.E. Wagenlehner ${ }^{\mathrm{a}}$ \\ ${ }^{a}$ Clinic for Urology, Paediatric Urology and Andrology, Justus-Liebig University of Giessen, Giessen, Germany; \\ bDepartment of Urology, Technical University of Munich, Munich, Germany; 'Saxonia R\&D, Radeberg, Germany; \\ dDepartment of Urology, Technical University of Dresden, Dresden, Germany
}

\section{Keywords}

Urinary tract infection - Catheter biofilm · Antiseptics

\begin{abstract}
Background: A catheter allowing a release of antibacterial substances such as antiseptics into the bladder could be a new way of preventing biofilm formation and subsequent catheter-associated urinary tract infections. Methods: Minimal inhibitory and bactericidal concentration (MIC/MBC) determinations in cation-adjusted Mueller-Hinton broth and artificial urine were performed for 4 antiseptics against 3 uropathogenic biofilm producers, Escherichia coli, Pseudomonas aeruginosa, and Proteus mirabilis. Furthermore, effects of octenidine and polyhexanide against catheter biofilm formation were determined by quantification of biofilm-producing bacteria. Results: Sodium hypochlorite showed MIC/ MBC values between 200 and $800 \mathrm{mg} / \mathrm{L}$ for all strains tested. Triclosan was efficient against $E$. coli and $P$. mirabilis (MIC $\leq 2.98 \mathrm{mg} / \mathrm{L}$ ) but ineffective against $P$. aeruginosa. Octenidine and polyhexanide showed antibacterial activity against all 3 species tested (MIC 1.95-7.8 and 3.9-31.25 mg/L). Both octenidine and polyhexanide were able to prevent biofilm
\end{abstract}

karger@karger.com www.karger.com/uin

Karger $\stackrel{\text { ' }}{5}$

GOPEN ACCESS
(C) 2021 The Author(s)

Published by S. Karger AG, Basel

This is an Open Access article licensed under the Creative Commons Attribution-NonCommercial-4.0 International License (CC BY-NC) (http://www.karger.com/Services/OpenAccessLicense), applicable to the online version of the article only. Usage and distribution for commercial purposes requires written permission. formation on catheter segments in a concentration dependent manner. Furthermore, adding $250 \mathrm{mg} / \mathrm{L}$ of each biocide disrupted biofilms formed by E. coli and P. mirabilis, whereas even $500 \mathrm{mg} / \mathrm{L}$ was not sufficient to completely destroy $P$. aeruginosa biofilms. Conclusion: Octenidine- and polyhexanide-containing antiseptics showed a broad effect against typical uropathogenic biofilm producers even in high dilutions. This study provides a basis for further investigation of the potential of octenidine and polyhexanide as prophylaxis or treatment of catheter biofilms.

(c) 2021 The Author(s)

Published by S. Karger AG, Basel

\section{Introduction}

Urinary tract infections (UTIs) are among the most common nosocomial infections and up to $90 \%$ of these are catheter associated. Indwelling catheters are a major risk factor for ascending UTIs and catheter-associated UTIs (CAUTIs) as well as other complications and are especially important in urology $[1,2]$. The most significant catheter complications are severe mechanical traumas such as perforation, partial urethral damage and uri- 
nary leakage, symptomatic bacterial infection, anaphylaxis, catheter toxicity, and hypersensitivity [3]. Especially CAUTIs are related to increased morbidity and mortality and costs such as increased length of stay and hospital costs [4]. Catheter-associated bacteriuria is associated with biofilm formation along the catheter surface [5]. Biofilms are surface-associated bacterial conglomerates that enable bacteria to survive and persist on abiotic and biotic surfaces. Biofilms are encased within a self-produced extracellular polymeric matrix which enables pathogens to escape host defences but also enhances antimicrobial resistances. The organisms that commonly contaminate indwelling urinary catheters and develop biofilms are Pseudomonas aeruginosa, Escherichia coli, Proteus mirabilis, Staphylococcus epidermidis, and other gram-negative bacteria $[6,7]$. A recent systematic review on CAUTIs revealed that there is no sufficient evidence to show a benefit of alternative type of catheters compared to standard catheters, or alternative catheter materials, or alternative antiseptic-impregnated catheters [8]. In addition there is no consensus on the best scientific approach, how to study CAUTIs, and preventive strategies. Comparative studies however have investigated how to best quantify catheter biofilm formation [9] or biofilm location on catheters [10]. Several evidencebased guidelines provide recommendations for the prevention of CAUTI including avoidance of catheter use, improving catheter design, or fabricating catheter coatings $[6,11]$. Nitrofurazone-coated catheters have been shown to decrease the risk for CAUTI development [12]. However, these catheters are accompanied with a more frequent removal and increased patient discomfort, as well as a possible emergence of antimicrobial resistance [12].

There is therefore a strong need for novel urinary catheter designs, aiming at decreasing complications, such as CAUTIs [3]. A catheter allowing a continuous release of antibacterial substances as antiseptics into the bladder could be a new way of preventing biofilm formation and subsequent catheter-associated UTIs [13]. Such a catheter could be used for biofilm prophylaxis by rinsing the bladder and the space between the catheter and the urethra, either intermittently or constantly with the antibacterial solution under development. Since antimicrobial resistance is a worldwide major public health problem, the usage of antibiotics should be prudent, thoughtful, and rational [14]. Hence, the undirected use of antibiotics as prophylactic therapy using the improved catheter should be avoided. As a replacement, antiseptics could be used as reservoir filling. A wide va- riety of active biocides contained in antiseptics have been used for hundreds of years for disinfection and preservation. Biofilm preventing or disrupting activity of some antiseptics could be shown before. Thus, cationic octenidine dihydrochloride (referred further as octenidine) inactivates Acinetobacter baumannii and Staphylococcus aureus biofilms formed on different surfaces including catheters $[15,16]$. Octenidine and polyhexanide reduced $P$. aeruginosa biofilms grown on polycarbonate slides [17]. In an in vitro urinary tract model, triclosan-impregnated urinary catheters were more effective in preventing the formation of $S$. aureus and $S$. epidermidis biofilms compared to nitrofural-treated catheters [18]. In addition, filling the retention balloon of urinary catheters with $10 \mathrm{~g} / \mathrm{L}$ triclosan in a catheterized bladder model prevented the biofilm formation of several uropathogens [19].

The objective of this study was to investigate the efficacy of the biocides octenidine, polyhexanide, triclosan, and sodium hypochlorite against the biofilm-producing uropathogenic species E. coli, P. aeruginosa, and P. mirabilis in artificial urine and the relevant concentrations needed. This should serve to assess the possible use of these agents as an antimicrobial filling for continuous release catheters as CAUTI prophylaxis.

\section{Materials and Methods}

Bacteria

From each species tested a reference strain (E. coli ATCC25922, $P$. aeruginosa ATCC27853, and P. mirabilis ATCC35659) as well as a clinical isolate from patients with UTI (E. coli UTI89, P. aeruginosa 568, and P. mirabilis CHD71) was used.

\section{Determination of Minimal Inhibitory and Bactericidal}

Concentrations (MIC/MBC)

The following disinfectants were tested: FARCO-fill ${ }^{\circledR}$ Protect (triclosan 3,000 mg/L; Farco-Pharma, Köln, Germany), LAVAN$\mathrm{OX}^{\circledR}(<0.08 \%$ sodium hypochlorite $<800 \mathrm{mg} / \mathrm{L}$; Serag Wiessner, Naila, Germany), Octenisept ${ }^{\circledR}$ (octenidine 1,000 mg/L; Schülke \& Mayr, Norderstedt, Germany), and Prontosan ${ }^{\circledR}$ (polyhexanide $1,000 \mathrm{mg} / \mathrm{L} ; \mathrm{B}$. Braun Melsungen AG, Melsungen, Germany). MIC and MBC determinations were described elsewhere [20]. All determinations were repeated thrice.

\section{Determination of Catheter Biofilm Preventing and Removing} Activities

Sterile latex catheters (Rüsch Gold; Teleflex Medical, Perak, Malaysia) were cut in $1 \mathrm{~cm}$ segments. These segments were placed in artificial urine with $1-10 \times 10^{8} \mathrm{CFU} / \mathrm{mL}$ of the tested bacteria. For biofilm-preventing assays, disinfectants were added in parallel to the bacteria and catheter segments were subsequently incubated for 1 day ( $P$. aeruginosa and P. mirabilis) or 3 days $\left(E\right.$. coli) at $37^{\circ} \mathrm{C}$ without shaking for biofilm formation. In case of the biofilm-re- 


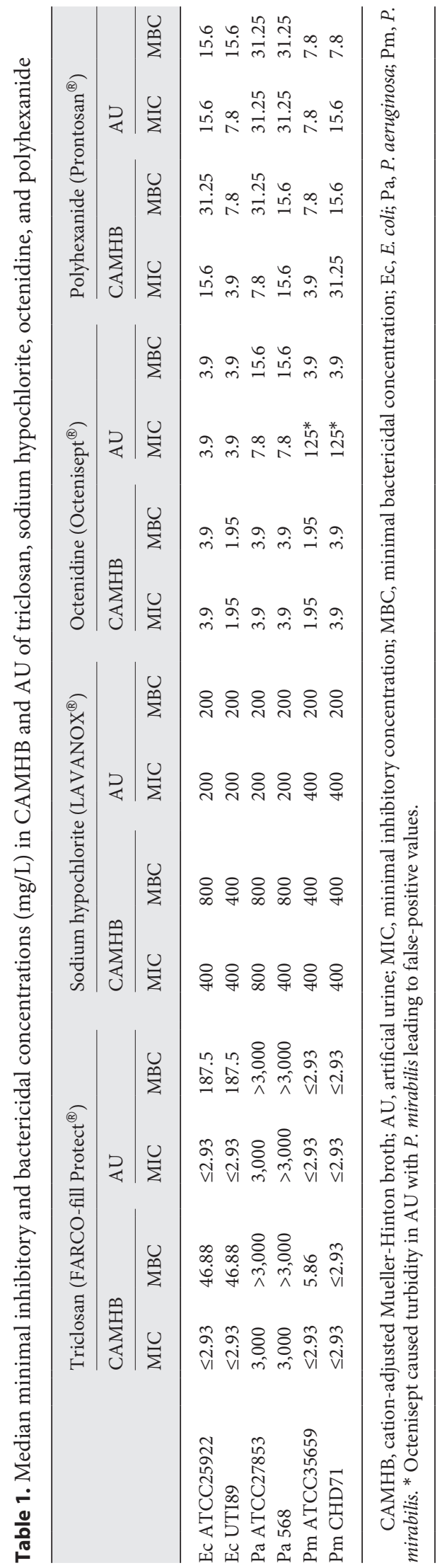

moving assays, biofilms were allowed to form before disinfectants were added and biofilms were quantified after further overnight incubation. For quantification of the planktonic bacteria, supernatants of the catheter segments were serial diluted in PBS and plated on cation-adjusted Mueller-Hinton broth (CAMHB) agar plates. For biofilm quantification, catheter segments were washed thrice in PBS. After placing them in $1 \mathrm{~mL}$ of PBS in $1.5-\mathrm{mL}$ reaction tubes, they were vortexed $1 \mathrm{~min}$ at full speed. Afterwards, sonification of the catheter segments was performed using ultrasonic bath Sonorex TK30. For E. coli biofilms, catheter segments were sonicated for $10 \mathrm{~min}$ [21]. Pseudomonas- and Proteus-treated catheter segments were sonicated twice for 5 min with a 2-min vortexing in between [22]. Determination of biofilm-forming CFU was carried out after another $1 \mathrm{~min}$ of vortexing step by serial dilution and plating on CAMHB agar plates. Data were plotted using GraphPad Prism 8.4.3. software (San Diego, CA, USA). Differences were identified following ANOVA and, when appropriate, Dunnett's test analysis of significance for each of the variables.

\section{Results}

\section{Determination of Inhibitory and Bactericidal Titres}

Four commercial disinfectants were used to test the inhibitory and bactericidal activity of the disinfecting agents triclosan (FARCO-fill ${ }^{\circledR}$ Protect), sodium hypochlorite $\left(\right.$ LAVANOX $\left.^{\circledR}\right)$, octenidine $\left(\right.$ Octenisept $\left.{ }^{\circledR}\right)$, and polyhexanide (Prontosan ${ }^{\circledR}$ ) against 3 uropathogenic species. FARCO-fill Protect containing a concentration of $3,000 \mathrm{mg} / \mathrm{L}$ triclosan showed almost no activity against $P$. aeruginosa, while it was highly bactericidal with MBC values of at least $5.86 \mathrm{mg} / \mathrm{L}$ against $P$. mirabilis. For E. coli, MIC values were below the tested minimum of $2.93 \mathrm{mg} / \mathrm{L}$ but $\mathrm{MBC}$ values ranged between 46.88 and $187.5 \mathrm{mg} / \mathrm{L}$ suggesting more bacteriostatic activity against these bacteria. Furthermore, it seemed to be less active in artificial urine compared to CAMHB (Table 1). MIC and MBC values for sodium hypochlorite ranged from 800 to 400 $\mathrm{mg} / \mathrm{L}$ and $400-200 \mathrm{mg} / \mathrm{L}$ for all tested strains in CAMHB and artificial urine, respectively. The treatment of $P$. $m i-$

Fig. 1. Activity of polyhexanide and octenidine against bacterial catheter biofilms. Polyhexanide and octenidine in different concentrations were added once at the beginning of the biofilm formation process to artificial urine containing bacteria and catheter segments $(\mathbf{a}, \mathbf{c})$ or biofilms formed on catheter segments in artificial urine were treated with $250 \mathrm{mg} / \mathrm{L}$ (E. coli and P. mirabilis) or 500 $\mathrm{mg} / \mathrm{L}$ ( $P$. aeruginosa) polyhexanide $(\mathrm{P})$ and octenidine $(\mathrm{O})$ for 24 h. Planktonic (c, d) and biofilm-forming bacteria (a, b) were quantified. Open symbols show the values of the individual measurements. Horizontal lines show median values. ctrl, control without adding disinfectants; LOD, limit of detection; nd, not determined. ${ }^{*} p<0.05$ over control, ${ }^{* *} p<0.01$ over control, $* * * p<$ 0.001 over control, $* * * * p<0.0001$ over control.

(For figure see next page.) 


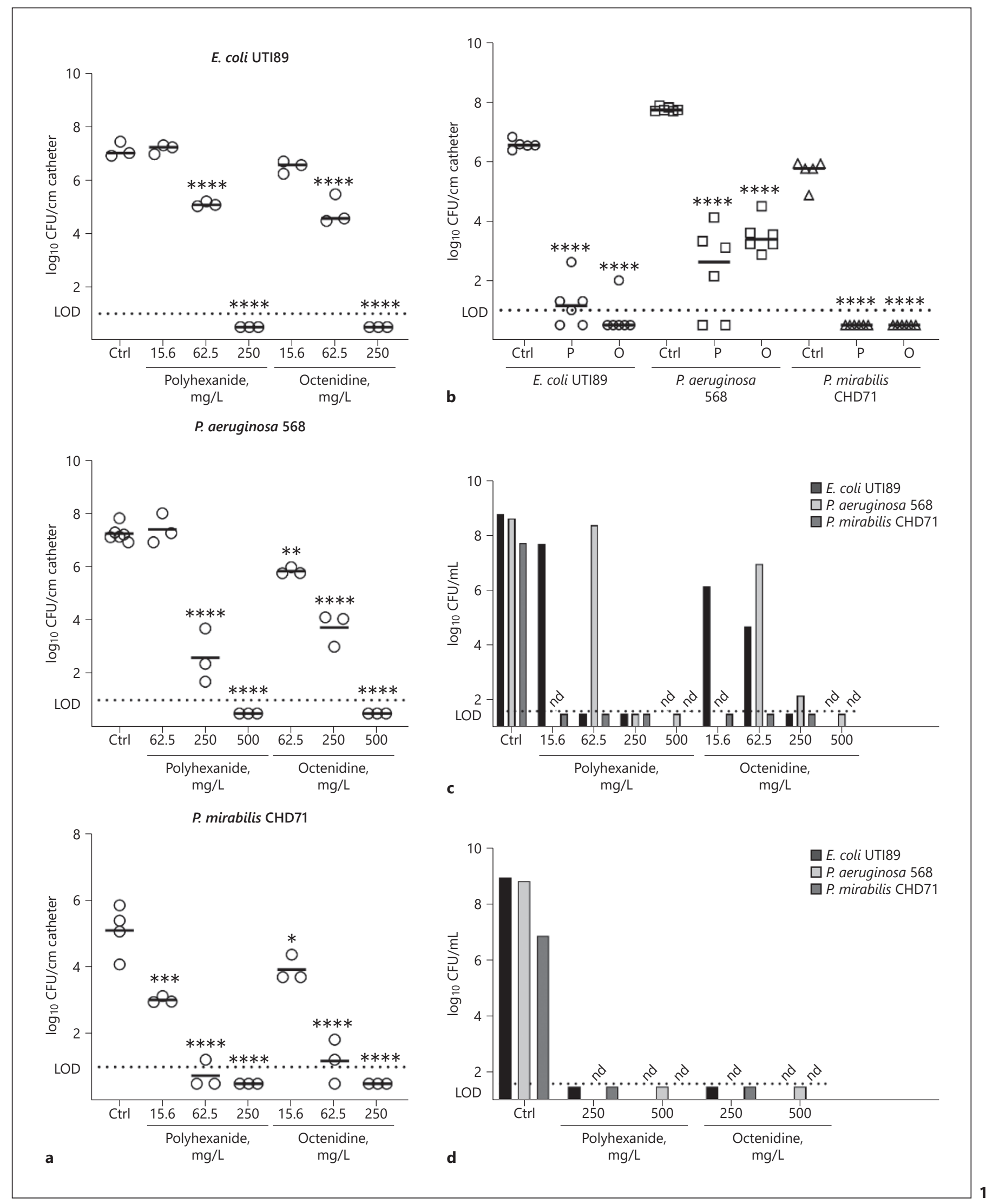

Anti-Biofilm Effects against 
rabilis with Octenisept ${ }^{\circledR}$ in artificial urine caused turbidity also in wells where no bacteria were found after plating. Due to these false positives, the MIC values appear to be higher than they really are. Based on the MBC values, it can be assumed that the MIC values for P. mirabilis in artificial urine were at least $3.9 \mathrm{mg} / \mathrm{L}$ (Table 1). MIC and $\mathrm{MBC}$ values of octenidine ranged between 1.95 and 3.9 $\mathrm{mg} / \mathrm{L}$ for $E$. coli and between 7.8 and $15.6 \mathrm{mg} / \mathrm{L}$ for $P$. aeruginosa, with a slight reduction of bactericidal activity in artificial urine for Pseudomonas. MIC values of polyhexanide ranged from 3.9 to $15.6,7.8-31.25$, and 3.9$31.25 \mathrm{mg} / \mathrm{L}$ for E. coli, P. aeruginosa, and P. mirabilis, respectively. $\mathrm{MBC}$ values were equal or 1-2 dilution steps lower (Table 1).

\section{Determination of Anti-Biofilm Activities}

In the next step, we were interested in the effectivity of octenidine and polyhexanide against bacterial catheter biofilms. Therefore, on the 1 hand the disinfectants were added during the process of biofilm formation on catheter segments to test their preventing activity. On the other hand, both were added after the biofilm has formed to determine the biofilm destructive potential. While $62.5 \mathrm{mg} / \mathrm{L}$ of polyhexanide killed all planktonic E. coli, biofilms on the catheter segments were still detectable but reduced. Using $62.5 \mathrm{mg} / \mathrm{L}$ of octenidine, reduced numbers of planktonic as well as biofilm bacteria were detected, compared to the untreated control. In contrast, using $250 \mathrm{mg} / \mathrm{L}$ of both biocides completely prevented biofilm formation (Fig. 1). In addition, $250 \mathrm{mg} / \mathrm{L}$ of octenidine as well as polyhexanide were able to almost completely disrupt the formed E. coli biofilm on the catheter segments (Fig. 1). For P. mirabilis $15.6 \mathrm{mg} / \mathrm{L}$ of both disinfecting agents were sufficient to kill all planktonic bacteria and to significantly reduce biofilm formation on catheter segments; 62.5 $\mathrm{mg} / \mathrm{L}$ almost completely abolished biofilm formation, whereas no biofilms were detected at all with $250 \mathrm{mg} / \mathrm{L}$ of polyhexanide as well as octenidine. In addition, formed $P$. mirabilis biofilms could be completely disrupted using $250 \mathrm{mg} / \mathrm{L}$ of both biocides (Fig. 1). Biofilms formed by $P$. aeruginosa were more robust against the disinfectants. Concentrations of $62.5 \mathrm{mg} / \mathrm{L}$ had almost no effect on the planktonic as well as biofilm bacteria for both biocides, whereas 250 and $500 \mathrm{mg} / \mathrm{L}$ killed the planktonic bacteria. In contrast to the Enterobacteriaceae, using $250 \mathrm{mg} / \mathrm{L}$ of polyhexanide or octenidine only reduced the numbers of biofilm $P$. aeruginosa. Here $500 \mathrm{mg} / \mathrm{L}$ were needed to completely prevent a biofilm formation. In addition, even incubation with $500 \mathrm{mg} / \mathrm{L}$ of both biocides was not able to completely destroy the formed biofilms (Fig. 1).

\section{Discussion}

Catheter-associated complications such as infections are very frequent [3]. Apart from avoiding urinary catheters or reducing catheter lengths, there is very little evidence showing a benefit for other preventive strategies, such as alternative type of catheters, different catheter materials, antiseptic impregnated catheters, or other measures [8]. There is therefore a strong need to reduce catheter-associated complications by evaluating novel catheter designs and preventive measures [3]. Despite the heterogeneous methodology used to assess and evaluate catheters and the involved biofilm formation, studies have shown how to best quantify biofilm formation and standardize the assessment of biofilm in and around a catheter [9], which was used in this study. $\mathrm{MIC} / \mathrm{MBC}$ determination of the 4 antiseptics tested revealed sodium hypochlorite (LAVANOX ${ }^{\circledR}$ ) as the least active substance against the 3 uropathogen species. Triclosan was bactericidal against $E$. coli and $P$. mirabilis but not against $P$. aeruginosa. Triclosan inhibits the fatty acid synthesis by binding to bacterial enoyl-acyl carrier protein reductase enzyme FabI. $P$. aeruginosa in contrast to the tested Enterobacteriaceae, encodes a second enoyl-acyl carrier protein reductase which is resistant to triclosan [23]. Antiseptics containing the biocides octenidine and polyhexanide showed the best activity against all 3 bacterial species tested. Furthermore, determination of the anti-biofilm activity showed that Octenisept ${ }^{\circledR}$ containing octenidine as well as Pronto$\operatorname{san}{ }^{\circledR}$ containing polyhexanide were able to prevent biofilm formation of E. coli, P. aeruginosa, and P. mirabilis in a concentration-dependent manner. Using the new developed catheter with slow release of these biocides into the bladder could possibly be used as biofilm prophylaxis. However, it is important to note that for a complete biofilm prevention, concentrations of at least $250 \mathrm{mg} / \mathrm{L}$ of both disinfecting agents were required. This corresponds to a maximum 1:4 dilution of the disinfectant, assuming an average urine production of $1.5 \mathrm{~L}$ per day will lead to a 1:25 dilution for a disinfectant released with $60 \mathrm{~mL} / 24 \mathrm{~h}$ from the catheter reservoir. Thus, only a reduction but no complete prevention of biofilm formation would be expected. However, our experimental design tested only a single-dosage treatment. A treatment approach using multiple doses while additional imitation of the bladder voiding would reflect a more accurate picture of the in vivo situation. Here, an increase of the anti-biofilm effectiveness and a reduction in the required concentration of the bio- 
cides would be expected. However, this still has to be proven in further experiments. In addition, safety issues have not been evaluated in this in vitro study. However previous studies with keratinocytes indicated that Prontosan is better tolerated than Octenisept for concentrations up to $175 \mathrm{mg} / \mathrm{L}$ [24]. Therefore, adding cell culture experiments to the experimental set-up could evaluate some of the safety issues. Therefore, the sensitivity of the bladder cells to the antiseptics and various concentrations has yet to be tested.

\section{Statement of Ethics}

Ethics approval was not required.

\section{Conflict of Interest Statement}

M.L., L.P., and M.W. declare no conflict of interest. K.G.N. reports personal fees from Adamed, Allecra, Apogepha, Bionorica, Enteris Biopharma, Galenus, GlaxoSmithKline, Hermes, Leo, Medice, MerLion, MSD Sharp \& Dohme, Paratek, Roche, Rosen, Saxonia, and Vifor outside the submitted work. F.M.E.W. reports personal fees and others from Achaogen, personal fees from AstraZeneca, personal fees from Bionorica, others from Enteris BioPharma, others from Helperby Therapeutics, personal fees from Janssen, personal fees from LeoPharma, personal fees from MerLion, personal fees from MSD, personal fees from OM Pharma/ Vifor Pharma, personal fees from Pfizer, personal fees from RosenPharma, personal fees and others from Shionogi, personal fees from VenatoRx, and personal fees from GSK outside the submitted work.

\section{Funding Sources}

This work was supported by CUP Laboratorien Dr. Freitag $\mathrm{GmbH}$.

\section{Author Contributions}

M.L. designed and carried out all experiments, performed the analysis, and wrote the manuscript in close cooperation with all coauthors considering their critical comments. K.G.N., L.P., M.W., and F.M.E.W. developed and initiated the project.

\section{References}

1 Sopeña-Sutil R, Medina-Polo J, Justo-Quintas J, Gil-Moradillo J, Garcia-Gonzalez L, Benítez-Sala R, et al. Healthcare-associated infections after lower urinary tract endoscopic surgery: analysis of risk factors, associated microorganisms and patterns of antibiotic resistance. Urol Int. 2018;100(4): 440-4.

2 Zhang JM, Liu J, Wang K, Zhang X, Zhao T, Luo HM. Observations of bacterial biofilm on ureteral stent and studies on the distribution of pathogenic bacteria and drug resistance. Urol Int. 2018;101(3):320-6.

3 Dellimore KH, Helyer AR, Franklin SE. A scoping review of important urinary catheter induced complications. J Mater Sci Mater Med. 2013;24(8):1825-35.

4 Canadian Patient Safety Institute, Canadian Institute for Health Information. Urinary tract infection. Hospital Harm Improvement Resource. Ottawa (ON): Canadian Patient Safety Institute; 2016 Apr: http://www. patientsafetyinstitute.ca/en/toolsResources/Hospital-Harm-Measure/Documents/ Resource-Library/HHIR\%20UTI.pdf Accessed 2018 Dec 30.

5 Stickler DJ. Bacterial biofilms in patients with indwelling urinary catheters. Nat Clin Pract Urol. 2008;5(11):598-608.
6 Nicolle LE. Catheter associated urinary tract infections. Antimicrob Resist Infect Control. 2014;3:23.

7 Niveditha S, Pramodhini S, Umadevi S, Kumar S, Stephen S. The isolation and biofilm formation of uropathogens in the patients with catheter associated urinary tract infections (CAUTI). J Clin Diagn Res. 2012;6: 1478-82.

8 Kranz J, Schmidt S, Wagenlehner F, Schneidewind L. Catheter-associated urinary tract infections in adult patients. Dtsch Arztebl Int. 2020;117(6):83-8.

9 Mandakhalikar KD, Rahmat JN, Chiong E, Neoh KG, Shen L, Tambyah PA. Extraction and quantification of biofilm bacteria: method optimized for urinary catheters. Sci Rep. 2018 May 23;8(1):8069.

10 Werneburg GT, Nguyen A, Henderson NS, Rackley RR, Shoskes DA, Le Sueur AL, et al. The natural history and composition of urinary catheter biofilms: early uropathogen colonization with intraluminal and distal predominance. J Urol. 2020 Feb;203(2): 357-64.

11 Singha P, Locklin J, Handa H. A review of the recent advances in antimicrobial coatings for urinary catheters. Acta Biomater. 2017;50:20-40.
12 Pickard R, Lam T, Maclennan G, Starr K, Kilonzo M, McPherson G, et al. Types of urethral catheter for reducing symptomatic urinary tract infections in hospitalised adults requiring short-term catheterisation: multicentre randomised controlled trial and economic evaluation of antimicrobial- and antiseptic-impregnated urethral catheters (the CATHETER trial). Health Technol Assess. 2012;16(47):1-197.

13 Vargas-Cruz N, Rosenblatt J, Reitzel RA, Chaftari A-M, Hachem R, Raad I. Pilot ex vivo and in vitro evaluation of a novel foley catheter with antimicrobial periurethral irrigation for prevention of extraluminal biofilm colonization leading to catheter-associated urinary tract infections (CAUTIs). BioMed Res Int. 2019;2019(3):1-10.

14 Abbo LM, Hooton TM. Antimicrobial stewardship and urinary tract infections. Antibiotics. 2014;3(2):174-92.

15 Narayanan A, Nair MS, Karumathil DP, Baskaran SA, Venkitanarayanan K, Amalaradjou MA. Inactivation of acinetobacter baumannii biofilms on polystyrene, stainless steel, and urinary catheters by octenidine dihydrochloride. Front Microbiol. 2016;7:847. 
16 Amalaradjou MA, Venkitanarayanan K. Antibiofilm effect of octenidine hydrochloride on staphylococcus aureus, MRSA and VRSA. Pathogens. 2014;3(2):404-16.

17 Harbs N, Siebert J. In vitro efficacy of octenidine and polihexanide against biofilms composed of Pseudomonas aeruginosa. GMS Krankenhaushyg Interdiszip. 2007;2:Doc45.

18 Gaonkar TA, Sampath LA, Modak SM. Evaluation of the antimicrobial efficacy of urinary catheters impregnated with antiseptics in an in vitro urinary tract model. Infect Control Hosp Epidemiol. 2003;24(7):50613.
19 Jones GL, Muller CT, O’Reilly M, Stickler DJ. Effect of triclosan on the development of bacterial biofilms by urinary tract pathogens on urinary catheters. J Antimicrob Chemother. 2006;57(2):266-72.

20 Loose M, Naber KG, Coates A, Wagenlehner FME, Hu Y. Effect of different media on the bactericidal activity of colistin and on the synergistic combination with azidothymidine against mcr-1-positive colistin-resistant Escherichia coli. Front Microbiol. 2020; 11:54.

21 Trautner BW, Darouiche RO, Hull RA, Hull S, Thornby JI. Pre-inoculation of urinary catheters with Escherichia coli 83972 inhibits catheter colonization by Enterococcus faecalis. J Urol. 2002;167(1):375-9.
22 Hola V, Peroutkova T, Ruzicka F. Virulence factors in proteus bacteria from biofilm communities of catheter-associated urinary tract infections. FEMS Immunol Med Microbiol. 2012;65(2):343-9.

23 Zhu L, Lin J, Ma J, Cronan JE, Wang H. Triclosan resistance of Pseudomonas aeruginosa PAO1 is due to FabV, a triclosan-resistant enoyl-acyl carrier protein reductase. Antimicrob Agents Chemother. 2010;54(2): 689-98.

24 Hirsch T, Koerber A, Jacobsen F, Dissemond J, Steinau HU, Gatermann S, et al. Evaluation of toxic side effects of clinically used skin antiseptics in vitro. J Surg Res. 2010;164(2):344-50 